\title{
BMJ Open Hip fracture evaluation with alternatives of total hip arthroplasty versus hemiarthroplasty (HEALTH): protocol for a multicentre randomised trial
}

\author{
Mohit Bhandari, ${ }^{1} \mathrm{P}$ J Devereaux, ${ }^{2}$ Thomas A Einhorn, ${ }^{3}$ Lehana Thabane, ${ }^{4}$ \\ Emil H Schemitsch, ${ }^{5}$ Kenneth J Koval, ${ }^{6}$ Frede Frihagen, ${ }^{7}$ Rudolf W Poolman, ${ }^{8}$ \\ Kevin Tetsworth, ${ }^{9}$ Ernesto Guerra-Farfán, ${ }^{10}$ Kim Madden, ${ }^{11}$ Sheila Sprague, ${ }^{12}$ \\ Gordon Guyatt, ${ }^{4}$ HEALTH Investigators
}

To cite: Bhandari $\mathrm{M}$, Devereaux PJ, Einhorn TA, et al. Hip fracture evaluation with alternatives of total hip arthroplasty versus hemiarthroplasty (HEALTH): protocol for a multicentre randomised trial. BMJ Open 2015;5:e006263.

doi:10.1136/bmjopen-2014006263

- Prepublication history for this paper is available online. To view these files please visit the journal online (http://dx.doi.org/10.1136/ bmjopen-2014-006263).

Received 13 August 2014 Revised 25 November 2014 Accepted 11 December 2014

CrossMark

For numbered affiliations see end of article.

Correspondence to Dr Mohit Bhandari; bhandam@mcmaster.ca

\section{ABSTRACT}

Introduction: Hip fractures are a leading cause of mortality and disability worldwide, and the number of hip fractures is expected to rise to over 6 million per year by 2050. The optimal approach for the surgical management of displaced femoral neck fractures remains unknown. Current evidence suggests the use of arthroplasty; however, there is lack of evidence regarding whether patients with displaced femoral neck fractures experience better outcomes with total hip arthroplasty (THA) or hemiarthroplasty (HA). The HEALTH trial compares outcomes following THA versus HA in patients 50 years of age or older with displaced femoral neck fractures.

Methods and analysis: HEALTH is a multicentre, randomised controlled trial where 1434 patients, 50 years of age or older, with displaced femoral neck fractures from international sites are randomised to receive either THA or HA. Exclusion criteria include associated major injuries of the lower extremity, hip infection(s) and a history of frank dementia. The primary outcome is unplanned secondary procedures and the secondary outcomes include functional outcomes, patient quality of life, mortality and hiprelated complications-both within 2 years of the initial surgery. We are using minimisation to ensure balance between intervention groups for the following factors: age, prefracture living, prefracture functional status, American Society for Anesthesiologists (ASA) Class and centre number. Data analysts and the HEALTH Steering Committee are blinded to the surgical allocation throughout the trial. Outcome analysis will be performed using a $\chi^{2}$ test (or Fisher's exact test) and Cox proportional hazards modelling estimate. All results will be presented with $95 \% \mathrm{Cls}$.

Ethics and dissemination: The HEALTH trial has received local and McMaster University Research Ethics Board (REB) approval (REB\#: 06-151).

Results: Outcomes from the primary manuscript will be disseminated through publications in academic journals and presentations at relevant orthopaedic conferences. We will communicate trial results to all participating sites. Participating sites will communicate

\section{Strengths and limitations of this study}

- This study has a large sample size of 1434 patients in order to detect small but important differences in outcomes.

- We are utilising an expertise-based randomised controlled trial design to help to eliminate differential performance and differential outcome assessments, and to reduce crossovers and ethical concerns associated with randomisation.

- The study has stringent methodological safeguards against bias including: use of a centralised randomisation system; blinding data analysts and the Steering Committee; standardisation and documentation; use of strategies to limit loss to follow-up; and adjudication of trial events by an independent Central Adjudication Committee.

- Surgeons and patients cannot be blinded to the surgical arms, leaving the assessment of outcomes and decisions to reoperate vulnerable to bias.

- We do not allow family members and friends to answer questionnaires on behalf of cognitively impaired patients; so some secondary outcomes will only apply to patients who are not cognitively impaired.

- The 2-year follow-up limits the study to shorter term outcomes.

results with patients who have indicated an interest in knowing the results.

Trial registration number: The HEALTH trial is registered with clinicaltrials.gov (NCT00556842).

\section{INTRODUCTION}

The number of hip fractures globally is expected to rise to over 6 million by the year 2050. ${ }^{12}$ Owing to projected ageing of the 
population, the number of hip fractures is likely to exceed 500000 annually in the USA and 88000 in Canada over the next 40 years. ${ }^{3-5}$ By the year 2040, the estimated annual healthcare costs associated with hip fractures will reach $\$ 9.8$ billion in the USA and $\$ 650$ million in Canada. ${ }^{6}$ Hip fractures account for more hospital days than any other musculoskeletal injury and represent more than two-thirds of all hospital days due to fractures. ${ }^{7}$ In the elderly, hip fractures are associated with a $30 \%$ mortality rate of 1 year postinjury, and temporary ${ }^{8}$ and often permanent impairment of independence and quality of life. ${ }^{9}{ }^{10}$ Worldwide, 4.5 million people are disabled from hip fractures yearly; the number of persons living with disability is expected to increase to 21 million in the next 40 years. ${ }^{135}$ The disability adjusted life-years lost as a result of hip fractures ranks in the top 10 of all causes of global disability. ${ }^{3}$

\section{Inconclusive clinical evidence}

Femoral neck fractures may be either non-displaced (ie, very little separation at the fracture site, about one-third of femoral neck fractures) or displaced (ie, greater separation at the fracture site, about two-thirds of femoral neck fractures). Orthopaedic surgeons often treat non-displaced fractures with internal fixation and displaced fractures with arthroplasty, which is also known as joint replacement.

We conducted a comprehensive literature search to identify all studies investigating total hip arthroplasty (THA; involves replacing the femoral head and the acetabulum) compared to hemiarthroplasty (HA; involves replacing the femoral head only) for displaced femoral neck fractures. We identified a recent meta-analysis of 14 studies ( $\mathrm{N}=1890$ patients). ${ }^{11}$ This meta-analysis demonstrated a lower risk of reoperation after THA compared with HA (relative risk $0.57,95 \%$ CI 0.34 to 0.96 ); however, this effect was mainly driven by randomised controlled trials (RCTs) without concealed treatment allocation. THA consistently resulted in better hip function (Harris Hip Score, weighted mean difference 5.4, 95\% CI 2.7 to 8.2) after follow-up intervals of $12-48$ months. ${ }^{11}$ We also identified two additional meta-analyses that provide comparisons of THA and HA. ${ }^{12}{ }^{13}$ Liao et $a l^{12}$ pooled RCTs of THA ( $\mathrm{N}=403$ patients) versus HA ( $\mathrm{N}=425$ patients), and found reductions in the risk of secondary procedures and wound infection with THA. Furthermore, Liao et al reported that the mobility rate in THA was better than in HA. Burgers $e t a l^{13}$ performed a meta-analysis of eight RCTs (N=986 patients) that suggested similar rates of secondary procedures, 1 year mortality, major complications and minor complications with THA and HA, but large benefits in patient function with THA. Despite the benefits that THA confers regarding functional outcomes, secondary procedures and infection rates, both meta-analyses reported higher hip dislocation rates with THA.

In a survey of 298 surgeons who were members of the Orthopaedic Trauma Association or affiliated with AO
International, 94-96\% of surgeons agreed that arthroplasty is the preferred treatment option in patients over 80 years of age with displaced femoral neck fractures. ${ }^{14}$ In addition, $73 \%$ of surgeons surveyed preferred HA to THA. ${ }^{14}$ In summary, current evidence suggests substantial benefits of THA over HA through decreased pain and improved function, but also increased rates of dislocation associated with THA, with similar rates of secondary procedures and mortality with the two procedures. Despite the apparent benefits of THA over HA, surgeons more often prefer HA to treat displaced femoral neck fractures. Previous trials comparing these two approaches were limited by methodological issues; therefore, the current trial aims to determine the effectiveness of THA compared to HA using a large sample size and high-quality methodology.

\section{Study objectives}

Our primary objective is to assess the impact of THA versus HA on rates of unplanned secondary procedures within 2 years for individuals with displaced femoral neck fractures.

Secondary objectives are:

1. To examine the effect of THA versus HA on health-related quality of life (Short Form-12, SF-12), functional outcomes and mobility (Western Ontario McMaster Osteoarthritis Index, WOMAC, and Timed Up and Go Test, TUG), and health outcome measures (EuroQol-5 Dimensions, EQ-5D)

2. To evaluate the effect of THA versus HA on mortality

3. To evaluate the effect of THA versus HA on hiprelated complications, including periprosthetic fracture, hip instability or dislocation, implant failure (loosening/subsidence and breakage), wound healing problems (including superficial/deep infection, wound necrosis), soft tissue problems (eg, pseudotumour), heterotopic ossification, abductor failure, implant wear and corrosion, osteolysis, neurovascular injury, decreased function and pain.

\section{METHODS AND ANALYSIS}

\section{Overview of study design}

HEALTH is a multicentre, concealed RCT of 1434 elderly patients who have sustained a displaced femoral neck fracture. We are using minimisation, a form of randomisation, to determine patient allocation. Surgeons across North America, Europe, Australia and Asia are participating. In conventional surgical hip fracture trials, all surgeons involved in the trial have performed both THA and HA based on the randomisation process. HEALTH utilises an expertise-based randomised trial design that allocates patients to surgeons with expertise in THA or HA (figure 1). Based on their expertise, surgeons perform either THA or HA. Surgeons who feel confident enough to perform both surgical strategies perform both THA and HA. 
Figure 1 Expertise-based randomised controlled trial. An expertise-based randomised controlled trial design randomises participants to surgeons with expertise in surgery $A$ or to surgeons with expertise in surgery $B$.

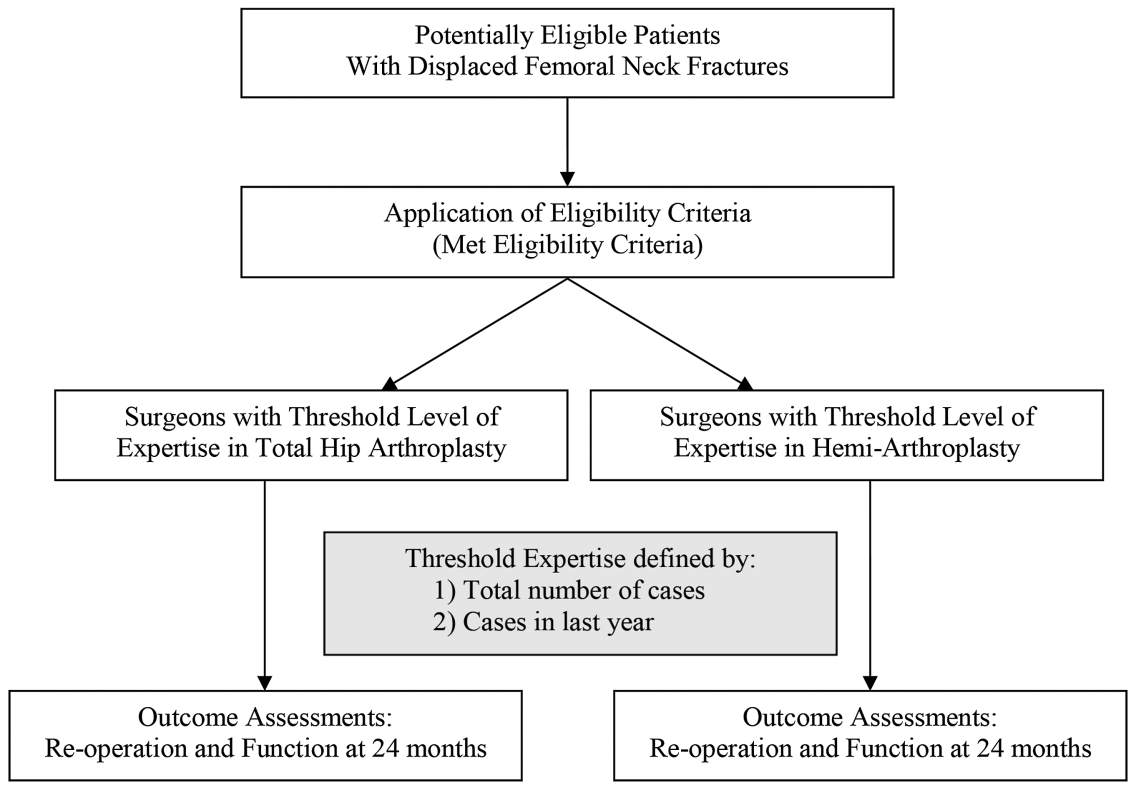

\section{Patient selection}

Eligibility criteria

The inclusion criteria are: (1) Adult men or women aged 50 years and older (with no upper age limit); (2) Fracture of the femoral neck confirmed with anteroposterior (AP) and lateral radiographs, or CT or MRI; (3) Displaced fracture that is not, in the judgment of the attending surgeon, optimally managed by reduction and internal fixation; (4) Operative treatment within $72 \mathrm{~h}$ of the patient being medically cleared for surgery; (5) Patient was ambulatory prior to fracture, though they may have used an aid such as a cane or a walker; (6) Anticipated medical optimisation for arthroplasty of the hip; (7) Provision of informed consent by patient or proxy; (8) Low energy fracture (defined as a fall from standing height); (9) No other major trauma (defined as an Injury Severity Score <17); and (10) Assurance that surgeons with expertise in both THA and HA are available to perform surgery.

The exclusion criteria are: (1) Patient not suitable for HA (eg, inflammatory arthritis, rheumatoid arthritis, pathological fracture (secondary to cancer) or severe osteoarthritis of the hip); (2) Associated major injuries of the lower extremity (eg, ipsilateral or contralateral fractures of the foot, ankle, tibia, fibula, knee or femur; dislocations of the ankle, knee or hip; or femoral head defects or fracture); (3) Retained hardware around the affected hip that will interfere with arthroplasty; (4) Infection around the hip (soft tissue or bone); (5) Patients with a disorder of bone metabolism other than osteoporosis (eg, Paget's disease, renal osteodystrophy, osteomalacia); (6) Patients with a previous history of frank dementia that would interfere with assessment of the primary outcome (ie, secondary procedures at 2 years); (7) Likely problems, in the judgement of the investigators, with maintaining follow-up (eg, patients with no fixed address, report a plan to move out of town, alcohol abuse issues or intellectually challenged patients without adequate family support); and (8) Patients whose fracture occurred as a result of an act of violence.

\section{Patient recruitment and screening}

The first patient was randomised into the vanguard phase of this trial on 21 January 2009. After a brief pause in enrolment between the vanguard and definitive phase, the first definitive patient was randomised on 8 October 2013. Enrolment is ongoing at the time of publication and is expected to be completed by December 2016. All patients presenting to participating surgeons with a diagnosed femoral neck fracture amenable to arthroplasty are screened for participation in the HEALTH trial. Such patients are classified as: (1) Excluded (if they do not meet the eligibility criteria); (2) missed (presumed eligible but missed due to error or staff availability); or (3) included (eligible and randomised). Study personnel obtain informed consent from all eligible patients. If a patient lacks capacity and is deemed unable to consent, study personnel may obtain informed consent from the patient's legally authorised representative and assent from the patient.

\section{Randomisation}

We are ensuring concealment of allocation by using a centralised $24 \mathrm{~h}$ computerised randomisation system that will allow internet-based randomisation. Patients are the unit of randomisation. To protect against prognostic imbalance between groups, we are using minimisation to ensure balance between intervention groups for several patient factors. The minimisation approach takes into account each preidentified prognostic variable and sums over the variables to allocate each patient to the treatment that will minimise the differences between groups for those prognostic variables. Unlike stratified 
randomisation, minimisation will work towards minimising the total imbalance for all factors taken together instead of considering mutually exclusive subgroups (strata). ${ }^{15}$ Based on our international survey of surgeons ${ }^{14}$ and current evidence, ${ }^{16}$ we are minimising for the following prognostic factors: (1) Age (ie, 50-80 years or greater than 80 years); (2) prefracture living setting (ie, institutionalised or not institutionalised); (3) prefracture functional status (ie, using aid or independent ambulator); (4) American Society for Anesthesiologists (ASA) Class (ie, Class I/II or III/IV/V); and (5) participating centre.

\section{Study interventions}

Total hip arthroplasty

In the THA group, we are not including minimally invasive THA (ie, 2 incision approaches) or hinged prostheses or capture cups. To optimise feasibility and applicability of results, we did not standardise the surgical approach, including the use of cemented components, the implant manufacturer or femoral head size. We are documenting the manufacturer, implant material and bearing surface of the implant.

\section{Hemiarthroplasty}

Surgeons use modern implants for HA, excluding nonmodular and non-canal filling unipolar implants, such as Moore's and Thompson's prostheses. We did not standardise the choice of modular unipolar versus bipolar HA nor whether implants are inserted with cement or with a press-fit design. We are documenting the manufacturer, implant material and bearing surface of the implant.

\section{Standardisation of procedures and perioperative care}

To ensure similar perioperative regimens, we recommend that participating centres standardise key aspects of preoperative and postoperative care. For preoperative care, we recommend standardisation of the following: (1) Preoperative antibiotic prophylaxis (eg, cephalosporins or equivalent coverage); (2) thromboprophylaxis (eg, oral anticoagulation drugs, thromboembolic deterrent stockings (TEDS), pneumatic compression boots or medical prophylaxis to be discontinued in sufficient time to allow for surgery as guided by international normalised ratio/partial thromboplasty time); and (3) medical consultation to optimise condition prior to surgery. For postoperative care, we recommend standardisation of the following: (1) Antibiotic prophylaxis (eg, cephalosporin or equivalent) for $24 \mathrm{~h}$; (2) thromboprophylaxis with unfractionated heparin, low-molecular-weight heparin $(\mathrm{LMWH})$, warfarin, antiplatelet agents or intermittent pneumatic compression boots; (3) weight-bearing as tolerated will be allowed as patients autoprotect the affected hip during rehabilitation. Postsurgery, patients are weight-bearing as tolerated and then advanced according to the attending surgeon's best judgment; (4) $600 \mathrm{mg}$ calcium by mouth daily and 1000 IU vitamin D per day (provided there are no contraindications), and further investigation and treatment of osteoporosis as recommended by a local osteoporosis expert/consultant; and (5) appropriate nutritional assessment with administration of oral micronutrient feeds as needed. Owing to a lack of evidence favouring a particular approach, we are recording but did not standardise the following: (1) Use of preoperative traction; (2) surgical delay; (3) type of anaesthetic (ie, general or regional); and (4) patient participation in physiotherapy and rehabilitation.

\section{Study outcomes}

Primary study outcome

The primary outcome is any unplanned secondary procedure within 2 years of the initial hip replacement surgery. A Central Adjudication Committee (CAC) will review each reported secondary procedure to determine that they are study events (ie, unplanned), and they will confirm the type of the procedure and the reason for the procedure (table 1 ).

\section{Secondary study outcomes}

Secondary outcomes include: (1) Functional outcome and quality of life measured using self-administered and interview-administered questionnaires. (2) The effect of THA versus HA on mortality; (3) hip-related complications including periprosthetic fracture, hip instability or dislocation, implant failure (loosening/subsidence and breakage), wound healing problems (including superficial/deep infection, wound necrosis), soft tissue problems (eg, pseudotumour), heterotopic ossification, abductor failure, implant wear and corrosion, osteolysis, neurovascular injury, decreased function or pain.

Functional outcome and quality of life are measured using self-administered and interview-administered questionnaires. Functional outcome questionnaires include a generic health status measurement instrument (SF-12), ${ }^{17}$ a hip function and pain questionnaire (WOMAC), ${ }^{18}$ a health outcome measure (EQ-5D) ${ }^{19}$ and a functional mobility test (TUG).$^{20}$

The SF-12 questionnaire is a self-administered, 12-item questionnaire that measures health-related quality of life in eight domains. Both physical and mental summary scores can be obtained. Each domain is scored separately from 0 (lowest level) to 100 (highest level). The instrument has been extensively validated and has demonstrated good construct validity, high internal consistency and high test-retest reliability. ${ }^{21}$ It is frequently used in orthopaedics for evaluating fracture outcomes.

The WOMAC index is self-administered and assesses the three dimensions of pain, disability and joint stiffness in knee and hip osteoarthritis using a battery of 24 questions. ${ }^{18}$ It is a valid, reliable and responsive measure of outcome and has been used in several studies involving a wide range of lower extremity conditions. ${ }^{22}$

The EQ-5D is a comprehensive, compact health status classification and health state preference system. ${ }^{19}$ This questionnaire is widely used, and has demonstrated validity and sensitivity in many populations. ${ }^{19}{ }^{21}$ 
Table 1 Classification of types and reasons for unplanned secondary procedures

\begin{tabular}{ll}
\hline Specific unplanned secondary procedures include & Classification of the reason for secondary \\
procedures is as follows & Treat a periprosthetic fracture \\
Open reduction of hip dislocation & Treat hip instability or dislocation \\
Open reduction of fracture & Treat infection-superficial \\
- Soft tissue procedure & Treat infection-deep \\
- Full implant exchange & Treat wound necrosis \\
Partial implant exchange-stem only & Treat another wound healing problem \\
Partial implant exchange-head only & Remove heterotopic ossification \\
Partial implant exchange-liner only & Manage abductor failure \\
Partial implant exchange-head and liner & Manage another soft tissue problem (ie, \\
Partial implant exchange-acetabular component only & pseudotumour) \\
- Implant adjustment-re-orientation of the stem & Correct implant failure-loosening or subsidence \\
Implant adjustment-re-orientation of the acetabulum & Correct implant failure-breakage \\
component & Treat implant wear \\
Explant removal with no replacement & Treat osteolysis \\
\hline Supplementary fixation & Treat implant corrosion \\
\hline
\end{tabular}

The EQ-5D is generalisable as it is widely used in Europe and will be useful for our definitive trial in which we will be including international sites.

The TUG involves observing the patient and documenting the time, ${ }^{20}$ in seconds, it takes for the patient to rise from a standard armchair, walk to a line on the floor $3 \mathrm{~m}$ away, turn, return and sits down again. The TUG has been used in many clinical contexts, including orthopaedics, ${ }^{23}$ rheumatology ${ }^{24}$ and for predicting geriatric falls. ${ }^{25}$

\section{Adjudication of study events}

The HEALTH CAC is comprised of five orthopaedic surgeons who specialise in hip surgery and have expertise in research methodology and experience with clinical trials. The CAC are reviewing: (1) Cases where fracture eligibility is in doubt; (2) postoperative X-rays to assess the technical placement of prostheses; (3) all reported secondary procedures and fracture-related complications to determine if the secondary procedure and/or fracture-related complication meets the requirements of the study criteria; and (4) cases of mortality to confirm the cause of death. All centres submit digital X-rays to the HEALTH Methods Centre. We post all relevant patient records devoid of personal identifiers (ie, case report forms and X-rays) on a specially designed, password-protected website for study adjudication. Adjudication occurs after patients have completed their 2 year follow-up. Any disagreements among the CAC members are resolved during conference calls. All decisions made by the Committee are final.

\section{Study follow-up}

All patients are followed for a period of 2 years. At each follow-up interval, patients' health status and outcomes are recorded. In addition, during the 2-year follow-up visit, the surgeon documents any secondary procedure that may be planned for the patient. Figure 2 shows the schedule of events and assessments at each time point.

Our choice of a 2-year follow-up period is dictated by two factors. First, previous studies have reported that $75 \%$ of revision surgeries occur before 12 months for patients treated with arthroplasty for displaced femoral neck fractures. ${ }^{26}$ Thus, we can expect that the majority of revision surgeries will occur within 2 years. Since increasing follow-up to beyond 2 years will yield little additional information on secondary procedure rates, efficient use of resources dictates a 2-year follow-up. Second, a full 2 years of follow-up will provide sufficient time to assess any potential gains in function and quality of life provided by either surgical alternative.

\section{Protecting against sources of bias Blinding}

While surgeons, patients and outcome assessors cannot be blinded to the surgical arms (ie, THA or HA), data analysts and the Steering Committee will remain blinded throughout the trial. Secondary procedures, the primary outcome, are objective and lack of blinding introduces minimal threats to validity. Additionally, the HEALTH trial design eliminates differential expertise bias by establishing a minimal threshold for experience, as well as ensuring that all surgeons, performing either THA or $\mathrm{HA}$, are dedicated to and have sufficient expertise with the procedure.

\section{Surgeon expertise}

Surgeons participating in the HEALTH trial are asked to meet both of the following two criteria of expertise for either THA or HA: (1) Perform at least 50 procedures 


\begin{tabular}{|c|c|c|c|c|c|c|c|c|c|c|c|c|c|}
\hline \multirow[b]{2}{*}{$\begin{array}{l}\text { Radiographs \& } \\
\text { Event Forms }\end{array}$} & \multicolumn{3}{|c|}{ Pre-Surgery } & \multirow{2}{*}{\begin{tabular}{|l} 
Surgery \\
(Day 0)
\end{tabular}} & \multicolumn{9}{|c|}{ Post Surgery } \\
\hline & Screening & Enrolment & Baseline & & $\begin{array}{l}\leq 48 \text { hrs } \\
\text { Post } \\
\text { Surgery }\end{array}$ & Discharge & $\begin{array}{l}1 \\
\text { wk }\end{array}$ & $\begin{array}{l}10 \\
\text { wk }\end{array}$ & $\begin{array}{l}6 \\
\mathrm{~m} 0\end{array}$ & $\begin{array}{l}9 \\
\text { mo }\end{array}$ & $\begin{array}{l}12 \\
\text { mo }\end{array}$ & $\begin{array}{l}18 \\
\text { mo }\end{array}$ & $\begin{array}{l}24 \\
\text { mo }\end{array}$ \\
\hline Patient Contact & & $\bullet$ & & & $\bullet$ & $\bullet$ & $\bullet$ & $\bullet$ & $\bullet$ & $\bullet$ & $\bullet$ & $\bullet$ & $\bullet$ \\
\hline Radiographs & & & $\bullet$ & & $\bullet$ & & & - & & & - & & - \\
\hline Screening & $\bullet$ & & & & & & & & & & & & \\
\hline Informed Consent & & $\bullet$ & & & & & & & & & & & \\
\hline Randomisation & & $\bullet$ & & & & & & & & & & & \\
\hline Baseline & & & - & & & & & & & & & & \\
\hline Surgical Report & & & & $\bullet$ & & & & & & & & & \\
\hline Post-Op Care & & & & & $\bullet$ & & & & & & & & \\
\hline $\begin{array}{l}\text { Clinic or } \\
\text { Telephone } \\
\text { Follow-Up }\end{array}$ & & & & & & & $\bullet$ & $\bullet$ & $\bullet$ & $\bullet$ & $\bullet$ & $\bullet$ & $\bullet$ \\
\hline $\begin{array}{l}\text { Secondary } \\
\text { Procedure }\end{array}$ & & & & & * & & * & * & * & * & * & * & $*$ \\
\hline Adverse Event & & & & $*$ & * & & * & * & * & $*$ & * & $*$ & $*$ \\
\hline Timed Up and Go & & & & & & & & $\bullet$ & $\bullet$ & & $\bullet$ & & - \\
\hline SF-12 & & & & & & & $\bullet$ & $\bullet$ & $\bullet$ & • & • & - & $\bullet$ \\
\hline WOMAC & & & & & & & $\bullet$ & $\bullet$ & $\bullet$ & $\bullet$ & $\bullet$ & $\bullet$ & $\bullet$ \\
\hline EQ-5D & & & & & & & $\bullet$ & $\bullet$ & $\bullet$ & - & $\bullet$ & $\bullet$ & - \\
\hline $\begin{array}{l}\text { Missed } \\
\text { Follow-Up }\end{array}$ & & & & & & * & * & $*$ & * & * & * & * & \\
\hline Early W/D & & & & & & * & * & * & * & * & * & $*$ & * \\
\hline
\end{tabular}

Figure 2 Schedule of events. ${ }^{*}$ Complete forms when and/or if applicable.

(either THA or HA) in their career (including residency experience in which they assumed responsibility for the procedure), and (2) perform at least five procedures per year. Surgeons who meet the threshold for both THA and HA will perform both procedures, if no overwhelming bias in favour of one procedure is evident. A surgeon is considered biased for an approach if they performed less than five cases of either procedure in their last 50 procedures for a displaced femoral neck fracture. Residents and fellows may perform the procedures under the supervision of a participating attending surgeon. The surgeon most responsible for the case must meet the threshold expertise criteria and must be present in the operating room for the critical aspects of the procedure (table 2). Our decision to set 50

Table 2 Critical aspects of operative procedure requiring presence of experienced surgeon

\section{Hemiarthroplasty}

- Trial component insertion and verification of hip stability

- Implant insertion to ensure correct version

- Cement procedure, if used

- Final assessment of hip stability after implant insertion

Total hip arthroplasty

- Trial component insertion and verification of hip stability

- Implant insertion to ensure correct alignment of femoral and acetabular components

- Cement procedure, if used

Final assessment of hip stability after implant insertion procedures as the threshold for experience is based on previous studies examining learning curves and respective outcomes in patients undergoing THA. ${ }^{27-29}$ Our survey of surgeons, who are members of the Orthopaedic Trauma Association (OTA) or European AO International trauma centres, suggests that the threshold of HA is likely similar or slightly lower, given the perception of increased difficulty of THA.

Devereaux $e t a l^{30}$ have outlined the advantages of this trial design which includes the following:

1. Elimination of differential expertise bias in which, in conventional designs, a larger proportion of surgeons are experts in one procedure under investigation over the other.

2. Differential performance, cointervention, data collection and outcome assessment are less likely than in conventional RCT.

With this approach, ethical concerns are reduced because all surgeries are conducted by surgeons with expertise and conviction in the concerned procedures. ${ }^{30}$

\section{Maximising patient retention}

Previous trials in hip fracture surgery have lost up to $50 \%$ of patients to follow-up. ${ }^{14}$ To avoid this problem, the strategies outlined in figure 3 are used to minimise the loss to follow-up. We have successfully used the majority of these strategies to maximise follow-up in other multicentre studies. Key features of this strategy include: (1) excluding individuals who are likely to present problems to the follow-up; (2) prior to hospital discharge, in addition to proving their own contact 


\begin{tabular}{|c|c|c|c|c|}
\hline $\begin{array}{l}\text { Before the study } \\
\text { begins }\end{array}$ & At screening & At baseline & During the study & $\begin{array}{c}\text { For patients who are } \\
\text { difficult to contact }\end{array}$ \\
\hline $\begin{array}{l}\text { - Create a study } \\
\text { identity that } \\
\text { includes a logo on } \\
\text { all study } \\
\text { documents } \\
\text { - Train research } \\
\text { personnel in } \\
\text { maintaining } \\
\text { participant } \\
\text { confidentiality } \\
\text { - Ensure all study } \\
\text { personnel are } \\
\text { aware of study } \\
\text { procedures }\end{array}$ & $\begin{array}{l}\text { - Exclude } \\
\text { participants who } \\
\text { are likely to be } \\
\text { difficult to follow } \\
\text { for the duration of } \\
\text { the trial (e.g. no } \\
\text { fixed addresss, } \\
\text { patients with } \\
\text { dementia and no } \\
\text { family support) } \\
\text { - Be explicit about } \\
\text { follow up } \\
\text { procedures } \\
\text { including when to } \\
\text { expect contact } \\
\text { from study staff, } \\
\text { how often, and } \\
\text { what type of } \\
\text { contact (in person, } \\
\text { email, phone etc.) }\end{array}$ & $\begin{array}{l}\text { - Collect several } \\
\text { personal contacts } \\
\text { including friends or } \\
\text { family members to } \\
\text { assist with locating } \\
\text { patients later } \\
\text { - Ensure informed } \\
\text { consent is } \\
\text { conducted in an } \\
\text { appropriate } \\
\text { manner, including } \\
\text { using examples and } \\
\text { explanations that } \\
\text { are accessible to a } \\
\text { lay audience }\end{array}$ & $\begin{array}{l}\text { - Provide } \\
\text { participants with a } \\
\text { choice of email, } \\
\text { phone, and/or in- } \\
\text { clinic visits when } \\
\text { appropriate (i.e. } \\
\text { when no } \\
\text { radiographs are } \\
\text { required) } \\
\text { - Be flexible on } \\
\text { scheduling in-clinic } \\
\text { visits } \\
\text { - Ensure participants } \\
\text { can easily contact } \\
\text { study personnel by } \\
\text { providing them } \\
\text { with contact } \\
\text { information that is } \\
\text { easy to access } \\
\text { - Routinely verify } \\
\text { that the } \\
\text { participant's } \\
\text { contact } \\
\text { information is up to } \\
\text { date }\end{array}$ & $\begin{array}{l}\text { - Try previously } \\
\text { disconnected } \\
\text { phone numbers } \\
\text { - Search online or in } \\
\text { telephone books } \\
\text { for updated } \\
\text { contact } \\
\text { information } \\
\text { - Search the } \\
\text { hospital's database } \\
\text { for hospital } \\
\text { admissions } \\
\text { - Try to contact } \\
\text { participants form a } \\
\text { different phone } \\
\text { number or at a } \\
\text { different time of } \\
\text { day } \\
\text { - Hold regular staff } \\
\text { meetings to } \\
\text { brainstorm creative } \\
\text { ways to locate } \\
\text { participants who } \\
\text { are dificult to } \\
\text { contact }\end{array}$ \\
\hline
\end{tabular}

Figure 3 Strategies to enhance follow-up rates.

information, each patient provides the name and address of alternate contacts who are likely to be aware of the patient's whereabouts; (3) patients receive a reminder for their next follow-up visit from the clinical research coordinator; and (4) follow-up visits coincide with standard fracture clinic visits. Additionally, patients can complete some study visits over the phone provided no radiographs are required.

\section{Minimising cointerventions and contamination}

Any patients who cross over from one treatment group to the other will be analysed in the group to which they were allocated, maintaining the intention to treat approach of the analysis. Surgical cointerventions, such as general, neurosurgical or orthopaedic procedures, performed in addition to the arthroplasty procedure have the potential to confound outcomes. The standardisation of key aspects of preoperative and postoperative care, together with the expertise-based trial design, will minimise the use of cointerventions in study patients. Clinical sites record the details of any major additional procedures performed, as well as the use of medications that affect bone, such as bisphosphonates, vitamin D, calcium, hormone replacement therapy, selective oestrogen receptor modulators, calcitonin and anabolic steroid therapy.

\section{Maximising protocol adherence}

Given the inherent variability in practice patterns among orthopaedic surgeons, we believe it is important to ensure that surgeons adhere as closely as possible to the surgical management protocol. The feasibility of this large study involving multiple centres further depends on minimal changes from current practice. Our study protocol closely follows currently accepted practice in the management of patients with femoral neck fractures. We, therefore, anticipate high compliance with our protocol.

\section{Statistical plan}

\section{Sample size determination}

The choice of sample size is based on a comparison of THA versus HA for the primary outcome (unplanned secondary procedures). All statistical hypotheses will be two-sided. $\alpha$ Levels of 0.05 for the primary and 0.01 for the secondary outcomes were chosen. Previous studies have reported secondary procedure rates in hip fracture patients treated with $\mathrm{HA}$ that have ranged from $4 \%$ to 
Table 3 Sample size calculations comparing total hip arthroplasty (THA) and hemiarthroplasty (HA)

\begin{tabular}{|c|c|c|}
\hline Study & $\begin{array}{l}\text { THA reoperation } \\
\text { rate }\end{array}$ & $\begin{array}{l}\text { HA reoperation } \\
\text { rate }\end{array}$ \\
\hline $\begin{array}{l}\text { van den Bekerom } \\
\text { et }\left.a\right|^{\beta 1}\end{array}$ & $5 / 11$ & $1 / 1$ \\
\hline ker et $\left.a\right|^{\beta 2}$ & & \\
\hline ldt $e t a l^{\beta 3}$ & & \\
\hline$a l^{\beta 4}$ & & \\
\hline Macaulay et a ${ }^{35}$ & $1 / 16(6.25)$ & $0 / 23$ \\
\hline \multicolumn{3}{|c|}{$\begin{array}{l}\text { Studies included in a meta-analysis comparing THA with HA for } \\
\text { the outcome of reoperation at } 1 \text { year. } \\
\text { THA versus HA combined effect: fixed and random, relative } \\
\text { risk=1.67 ( } 95 \% \mathrm{Cl} 0.86 \text { to } 3.24), \mathrm{p}=0.13 \text {. } \\
\text { Pooled event rates: THA fixed and random, incidence rate }=7.8 \% \\
\text { (95\% } \mathrm{Cl} 5.2 \text { to } 11.6) \text {. } \\
\mathrm{HA} \text { fixed, incidence rate }=5.3 \%(95 \% \mathrm{Cl} 3.2 \text { to } 8.9) \text {. } \\
\text { HA random, incidence rate }=4.9 \%(95 \% \mathrm{Cl} 2.6 \text { to } 9.2) \text {. }\end{array}$} \\
\hline
\end{tabular}

$10 \%$ within 1 year, with a weighted pooled risk of $5.3 \%$ $(95 \%$ CI $3.2 \%$ to $8.9 \%)$ in a fixed effect meta-analysis or $4.9 \%(95 \%$ CI $2.6 \%$ to $9.2 \%)$ using random effects. ${ }^{13}$ A pooled estimate from five randomised trials comparing THA with HA gave a relative risk of 1.67 (95\% CI 0.86 to $3.24, \mathrm{p}=0.13$; table 3$) .^{13} 31-35$

The sample size calculation reflects the proposed approach to the primary analysis, which will use the Cox proportional hazards model. The calculation is based on methods described by Collett. ${ }^{36}$ The goal is to calculate the required number of patients that will yield a sufficient number of outcome events (secondary procedures) in order to have adequate statistical power for a given size of treatment effect. This was performed taking into account the anticipated secondary procedure rates in the HA group, postulated values of the relative risk increase associated with THA versus HA, and the rates of mortality and loss to follow-up. Since some of these inputs are expected to change over the 2-year period of follow-up, the expected number of person-years of follow-up and the expected numbers of study events in each group were calculated, initially for the first year of follow-up; the calculation was then repeated for the second year of follow-up, after having estimated the number of patients in each group who would survive, be event-free and available for continued follow-up between 12 and 24 months postrandomisation.

Based on aggregate data from the pilot study, annual mortality rates of $15 \%$ and a loss to follow-up of $5 \%$ in each group were assumed, these rates applying to each of the 2 years of follow-up. Informed by the meta-analysis, the following assumptions were made: a 1 year risk of having a secondary procedure of $5 \%$ in the HA group and a corresponding risk of $1 \%$ in the second year. Various values of the relative risk reduction (HA vs THA) were then used to identify the specific value that would correspond to a cumulative risk difference between THA and HA of 5\% after 2 years. This figure was identified in a survey of participating surgeons as the minimally important difference to clinicians (unpublished data). Annual event risks by group were converted into equivalent hazard rates, assuming for simplicity that the hazard rate would be approximately constant during each of the 2 years. It is estimated that approximately $72 \%$ of the group receiving THA and $76 \%$ of the group receiving HA will be event-free and available for further follow-up at the start of the second year. The sample size was increased to allow for a combined $7.6 \%$ crossover rate from the assigned to the alternate treatment, based on pilot data. These assumptions lead to a required total sample size of 1316 patients, which will yield an expected number of 96 secondary procedures. The associated relative risk reduction (RRR) is 0.45 . These calculations were repeated after replacing the $5 \%$ event risk at 1 year for HA by $4 \%$ and $6 \%$ and leaving the other factors unchanged (table 4). To account for potential surgeon level effects, the sample size has been further increased by $9 \%$ to 1434 .

For the secondary outcomes, an important difference in SF-12 is considered to correspond to a moderate effect, as reported by Cohen, and to a minimally important difference in the SF-12 as reported by Ware. ${ }^{37}{ }^{38} \mathrm{In}$ both cases, the value is at least half the $\mathrm{SD}$, equivalent to 4-point difference in score. Specifying $\alpha$ level $=0.05$, $\beta=0.20$ (study power $=80$ ) and a SD of $8,{ }^{39}$ a sample of at least 128 patients (64 per group) is required to ensure detection of a half SD improvement. In clinical drug trials, a 9-point change in WOMAC functional score was accepted as a minimally significant improvement in symptoms. ${ }^{40}$ In this study, at least 90 patients (45 per group) would be required to detect this difference ( $\alpha$ level $=0.05, \beta=0.20, \sigma=15$ ). Previous studies have found a SD of 0.20 for the EQ-5D. ${ }^{41}$ To detect a difference of 0.10 (half the SD) with $80 \%$ power at $\alpha$ level of 0.05 , this study requires a total of 128 patients (64 per group). Thus, in all circumstances, the desired sample size of 1434 patients will be sufficient to detect clinically meaningful differences in the secondary measures of outcome.

\section{Primary analyses}

All outcome analyses will be performed by an intentionto-treat approach. To evaluate the effect of THA versus HA on time to unplanned secondary procedures (the

Table 4 Sample size calculations comparing total hip arthroplasty (THA) and hemiarthroplasty (HA)

\begin{tabular}{llcl}
\hline $\begin{array}{l}\text { HA 1 year } \\
\text { event risk }\end{array}$ & $\begin{array}{l}\text { Total patients } \\
\text { required }\end{array}$ & $\begin{array}{l}\text { Expected } \\
\text { events }(\mathbf{n})\end{array}$ & RRR \\
\hline $4 \%$ & 1123 & 72 & 0.50 \\
$5 \%$ & 1316 & 96 & 0.45 \\
$6 \%$ & 1435 & 108 & 0.42
\end{tabular}

Sample size requirement for HEALTH Pivotal Trial (non-inferiority design).

RRR, relative risk reduction 
primary outcome), a Cox proportional hazards model will be used with the following covariates: (1) Age (ie, $50-80$ years or $>80$ years); (2) prefracture living setting (ie, institutionalised or not institutionalised); (3) prefracture functional status (ie, using aid or independent ambulator); (4) American Society for Anesthesiologists (ASA) Class (ie, Class I/II or III/IV/V); and (5) participating centre. Results will be reported as HRs with $95 \%$ CIs. Kaplan-Meier curves will be constructed.

\section{Secondary analyses}

In the secondary analysis, we will adjust for additional baseline factors when examining the effect of THA versus HA on time to unplanned secondary procedures (the primary outcome). This will be a Cox proportional hazards model which will include the same variables that are included in our primary analysis and additionally adjust for (1) gender, (2) surgical approach and (3) head size, by including these as independent variables. Results will be reported as HRs with 95\% CIs.

A generalised linear model will estimate the effect of THA versus HA on quality of life (SF-12), function (WOMAC), health outcome (EQ-5D), and mobility (TUG) at follow-up using the following covariates that are included in our minimisation procedure: (1) Age (50-80 years or greater than 80 years); (2) prefracture living setting (ie, institutionalised or not institutionalised); (3) prefracture functional status (ie, using aid or independent ambulator); (4) ASA Class (ie, Class I/II or III/IV/V); and (5) participating centre.

Cox proportional hazards modelling will estimate the relative effect of THA versus HA on time to mortality and hip-related complications. Results will be reported as HRs with 95\% CIs. Kaplan-Meier curves will be constructed.

\section{Interim analyses}

The approach to interim analyses is guided by a desire to avoid spuriously inflated estimates of treatment effect. ${ }^{42}$ A single interim analysis will be performed when $60 \%$ of the planned patient-years of follow-up have been accrued. The data analyst will present the results of these analyses to our independent Data Monitoring Committee. The committee will be guided by the O'Brien-Fleming stopping rule based on the primary outcome, which will maintain the overall specified type I error rate at 5\% for the combined interim and final analyses. According to this rule, the required $\mathrm{p}$ value to declare a significant result in the interim analysis is 0.007 and at the final analysis, the required $p$ value is 0.047 . The rule is conservative, making it difficult to stop the trial early unless a large treatment effect is observed. We will only apply our stopping rule to the primary outcome. The secondary functional outcomes may demonstrate significance quickly due to the nature of the instruments, but we will not stop the study for that reason. No one other than committee members will be aware of the data on which the committee makes its decision and no one involved in the study will be aware of the content of their deliberations.

\section{Data management}

The case report forms (CRFs) are the primary data collection tool for the study. An Electronic Data Capture system (iDataFax) is being used to submit data to the Methods Centre located at McMaster University. On receipt of the data, the personnel at the Methods Centre make a visual check of the data and they query all missing data, implausible data and inconsistencies.

\section{Sensitivity analyses}

We will perform some sensitivity analyses to assess the robustness of the primary results. First, we will perform a competing risk analysis using Fine and Gray method to account for death as a competing risk. ${ }^{43}$ Second, we will use the random-effects analysis with a centre as a random-effect to account for the possibility of the centre effect or clustering within a centre. Lastly, based on the pilot results, we anticipate some crossovers during the trial-we will monitor these and perform some sensitivity analysis to assess their impact. All analyses will be performed using SAS V.9.2 (Cary, North Carolina, USA).

\section{Missing data}

Patients who are lost or withdrawn from the study prior to the full 24 month follow-up will be censored at their last visit where evaluation of the primary end point was completed.

\section{Data monitoring committee}

The purpose of the Data Monitoring Committee (DMC) is to advise the HEALTH Investigators regarding the continuing safety of study participants. The DMC consists of the Chair, who is an orthopaedic surgeon, one biostatistician and two additional orthopaedic surgeons. All members are independent of the trial investigators, and have neither financial nor scientific conflicts of interest with the trial. Further details regarding the DMC can be found in the DMC Charter, available from the corresponding author.

\section{ETHICAL CONSIDERATIONS}

All patients (or their legally authorised representative) included in this study will sign a consent form that describes this study and provides sufficient information for patients to make an informed decision about their participation. All participating centres will submit the consent form with the protocol for review and approval by their local research ethics board (REB), or institutional review board (IRB) for the study. Centres will obtain written consent from every patient, using the REB/IRB-approved consent form, before that patient undergoes any study procedure. Any amendments to the study protocol which may affect the conduct of the study, or the potential safety of or benefits to patients 
will require a formal amendment to the protocol requiring approval by McMaster University's REB and local research ethics boards for clinical sites. Any protocol amendments will be communicated to investigators, $\mathrm{REB} / \mathrm{IRB}$, trial participants and trial registries, as deemed necessary.

Information about study patients will be kept confidential and will be managed in accordance with the following rules: (1) All study-related information will be stored securely at the clinical site; (2) all study patient information will be stored in locked file cabinets and be accessible only to study personnel; (3) all CRFs will be identified only by a coded patient number and initials; (4) all records that contain patient names or other identifying information will be stored separately from the study records and can be identified only by the coded patient number and initials; and (5) all local databases will be password protected.

\section{DISSEMINATION}

Results from the primary manuscript will be submitted for publication regardless of whether or not there are significant findings. Every attempt will be made to ensure that the amount of time between completion of data collection and release of study findings are minimised.

Only the Methods Centre will have access to the full trial data set. Data for the primary publication will be analysed exclusively by the Methods Centre. Requests for access to the full trial data set for secondary publications are encouraged and can be initiated through a written request to Methods Centre personnel.

\section{DISCUSSION}

Previous orthopaedic trials have addressed the effect of THA versus HA on patient outcomes; however, the trials conducted to date are limited by small sample sizes, lack of concealed randomisation, differential expertise biases $^{12}$ and most were single centre initiatives that lacked sufficient power to inform surgical practice. Orthopaedic surgeons appear to currently favour HA, although current evidence suggests superior patientimportant outcomes with THA. ${ }^{13} 30$ The HEALTH trial will aim to resolve these controversies by establishing the effectiveness of each method of arthroplasty. This will have important clinical implications as each treatment is easily applicable and already in use in orthopaedic practice.

The HEALTH trial will address and overcome many of the limitations of previous orthopaedic hip fracture trials. First, the study sample size of 1434 patients is sufficient to detect small but important differences in outcomes and will ensure that our study objectives are met. Second, using an expertise-based RCT has many advantages over traditional orthopaedic hip studies, including eliminating differential performance and differential outcomes assessment, and reducing the number of crossovers and ethical concerns associated with randomisation. ${ }^{12}$ Furthermore, the HEALTH trial has stringent methodological safeguards against bias including: the use of a centralised system to randomise patients; blinding data analysts and the Steering Committee; standardisation and documentation of preoperative care, perioperative care and postoperative care; the use of strategies to limit loss to follow-up and adjudication of trial events by an independent CAC.

A key limitation of the HEALTH trial is that surgeons and patients cannot be blinded to the surgical arms. This leaves the assessment of outcomes and decisions to re-operate vulnerable to bias. We have limited this bias by using an objective primary outcome (unplanned secondary procedures) and by centrally adjudicating all primary outcome events using a group of independent orthopaedic surgeons. Additionally, we do not allow family members or friends to answer questionnaires on behalf of cognitively impaired patients; thus, some secondary outcomes will only apply to patients who are not cognitively impaired. Our trial is limited to shorter term outcomes-up to 2 years following the initial surgery. Therefore, our results may not apply when considering longer term outcomes.

The results of the HEALTH trial will be an important contribution to orthopaedic surgical literature and are likely to lead to changes in orthopaedic practice. Identifying the optimal approach to arthroplasty has the potential to improve the lives of hundreds of thousands of patients and to reduce the economic burden associated with hip fractures. If this trial shows that one approach is superior to the other, it will revolutionise the treatment of hip fractures globally and may potentially lead to the establishment of new clinical guidelines for treatment of displaced femoral neck fractures. In addition to the clinical impact, the HEALTH trial also has the potential to impact orthopaedic trial conduct. With this trial we will build collaborative relationships among countries and between clinical centres. This trial will also contribute to challenging the dogma that surgical trials are doomed to be confined to small single centre initiatives.

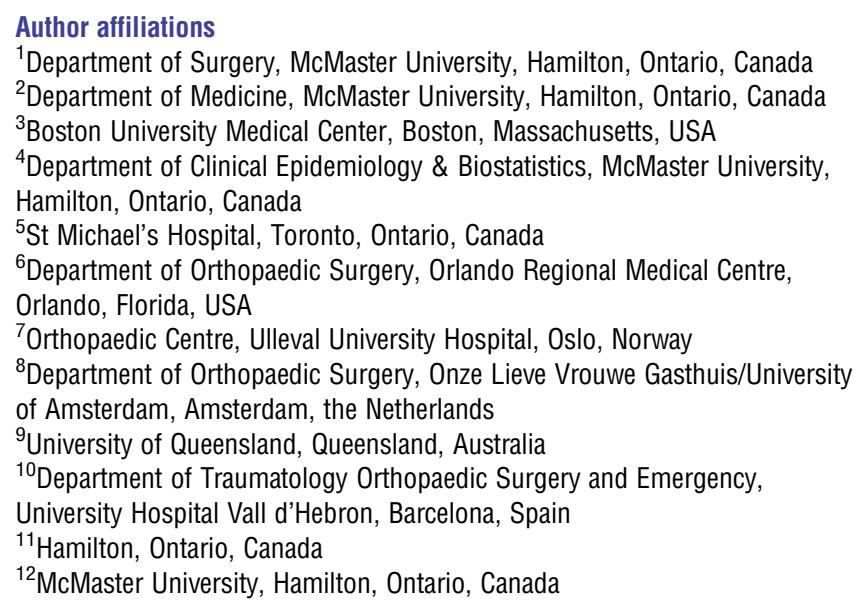

${ }^{12}$ McMaster University, Hamilton, Ontario, Canada 
Collaborators HEALTH Investigators: The following persons participated in the HEALTH trial. Canada: University of Calgary, Foothills Medical CentreRob Korley, Richard Buckley, Paul Duffy, Shannon Puloski, Kimberly Carcary, Melissa Lorenzo, Georgia Carstensen, Ross McKercher. University of Calgary, Rockyview General Hospita-Kelly Johnston, Greg Abelseth, Raul Kuchinad, Saboura Mahdavi. St. Michael's Hospital-Emil H Schemitsch, Michael D McKee, Jeremy A Hall, Aaron Nauth, Daniel Whelan, Timothy R Daniels, Earl R Bogoch, James P Waddell, Henry Ahn, Milena R Vicente, Jennifer T Hidy, Melanie T MacNevin. Sunnybrook Health Sciences Centre-Hans Kreder, Terry Axelrod, Richard Jenkinson, Markku Nousiainen, David Stephen, Veronica Wadey, Monica Kunz, Katrine Milner, Ria Cagaanan, Melanie MacNevin. Vancouver General Hospital-Peter J O'Brien, Piotr A Blachut, Henry M Broekhuyse, Pierre Guy, Kelly A Lefaivre, Gerard P Slobogean, Raman Johal, Irene Leung. Queen Elizabeth II Health Sciences Centre-Chad Coles, Ross Leighton, C Glen Richardson, Michael Biddulph, Michael Gross, Michael Dunbar, J David Amirault, David Alexander, Catherine Coady, Mark Glazebrook, David Johnston, William Oxner, Gerald Reardon, Ivan Wong, Kelly Trask, Shelley MacDonald. Memorial University of Newfoundland-Andrew Furey, Craig Stone, Minnie Parsons. University of British Columbia/Fraser Health Authority - Trevor Stone, Mauri Zomar, Robert McCormack, Kelly Apostle, Dory Boyer, Farhad Moola, Bertrand Perey, Darius Viskontas, Karyn Moon, Raely Moon. Hôpital du Sacré-Coeur de Montréal-Yves Laflamme, Benoit Benoit, Pierre Ranger, Michel Malo, Julio Fernandes, Karine Tardif, Julie Fournier. Hôpital Maisonneuve-Rosemont-Pascal André Vendittoli, Vincent Massé, Alain G Roy, Martin Lavigne, Daniel Lusignan. USA: Colorado Orthopaedic Consultants - Craig Davis, Philip Stull, Stewart Weinerman, Peter Weingarten, Steven Lindenbaum, Michael Hewitt, Rebecca Danielwicz, Janell Baker, Rubin Institute for Advanced Orthopaedics-Michael Mont, Donald E Delanois, Bhaveen Kapadia, Kimona Issa, and Marylou Mullen, Mayo ClinicAndrew Sems, Barb Foreman, Rothman Institute — Javad Parvizi, Tiffany Morrison, Orthopaedic Associates of Hartford - Courtland Lewis, Stephanie Caminiti, Boston University Medical Centre - Thomas A Einhorn, Paul Tornetta III, William R Creevy, Heather Silva, Michelle J Lespasio, Hope Carlisle, Lahey Clinic-Andrew Marcantonio, Michael Kain, Lawrence Specht, and John Tilzey, John Garfi, University of Pennsylvania-Samir Mehta, John L Esterhai Jr, Jaimo Ahn, Derek Donegan, Annamarie Horan, Kelly McGinnis, Emory University School of Medicine-James Roberson, Thomas Bradbury, Greg Erens, Kyle Webb, Indiana University—Brian Mullis, Karl Shively, Andrew Parr, Janos Ertl, Ripley Worman, Mark Webster, Judd Cummings, Valda Frizzell, Molly Moore, Orthopaedic Associates of Michigan—Clifford B Jones, James R Ringler, Debra L Sietsema, Jane E Walker, Texas Tech University— Enes Kanlic, Amr Abdelgawad, Juan Shunia, Mission Hospital Research Institute - Charles DePaolo, Susan Sutherland, Rachel Alosky, Duke University Medical Centre—Robert Zura, Maria Manson, Park Nicollet Institute-Gregg Strathy, Kathleen Peter, Paul Johnson, and Meaghan Morton, St. Elizabeth Health Centre-James Shaer, Tyson Schrickel, Barbara Hileman, Marina Hanes, Elisha Chance. Texas Institute for Hip and Knee Surgery-E Matthew Heinrich, David Dodgin, Michele LaBadie, University of California IrvineDavid Zamorano, Martin Tynan, Ran Schwarzkopf, John A Scolaro, Ranjan Gupta, Samuel Bederman, Nitin Bhatia, Bang Hoang, Douglas Kiester, Neil Jones, Gregory Rafijah, Damon Alavekios, Jason Lee, Akshay Mehta, Steven Schroder, Tom Chao, Vincent Colin, Phuc (Phil) Dang, Stephen Keun Heng, Gregory Lopez, Samuel Galle, Sohrab Pahlavan, Duy L Phan, Minal Tapadia, Christopher Bui, Nickul Jain, Tyler Moore, Nathan Moroski, Deeba Pourmand. University of Utah -Erik N Kubiak, Jeremy Gililland, David Rothberg, Christopher Peters, Christopher Pelt, Ami R Stuart, Kirby Corbey. Marshall University - Franklin D Shuler, James Day, Tigran Garabekyan, Felix Cheung, Ali Oliashirazi, Jonathon Salava, Linda Morgan, Timothy Wilson-Byrne, and Mary Beth Cordle. Allegheny Hospital-Timothy J Sauber, Edward Westrick, Robert Duerr, Michael Maher, Traci Salopek. University Orthopaedic Associates-Carlos A Sagebien, David A Harwood, Stephen Kayiaros, Patricia Seuffert. State University of New York at Buffalo (UBMD Orthopaedics and Sports Medicine)—Sridhar Rachala, Mark Anders, Christopher Mutty, Matthew Philips, Allison Cornwall, Mary Bayers-Thering. University of Arizona —Michael Dohm, Cindy Fastje. Netherlands: Amphia Ziekenhuis-Leon HGJ Elmans, Joost AAM van den Hout, Adrianus JP Joosten, Ad FA van Beurden, Stefan BT Bolder, Denise Eygendaal, Adrianus FCM Moonen, Rutger Cl van Geenen, Eric A Hoebink, Robert Wagenmakers, Wouter van Helden; Deventer Ziekenhuis-Hans-Peter W van Jonbergen, Herbert Roerdink, Joost M
Reuver, Alexander FW Barnaart, Elvira R Flikweert; Diaconessenhuis LeidenRover Krips, J Bernard Mullers, Hans Schüller; Flevoziekenhuis-Mark LM Falke, Frans J Kurek, Adrianus CH Slingerland; Gelderse Vallei-Jan P van Dijk, Wouter H van Helden; Gelre Ziekenhuizen-Hugo W Bolhuis, Pieter HJ Bullens, Mike Hogervorst, Karin E de Kroon, Rob H Jansen, Ferry Steenstra, Eric EJ Raven; IJsselland Ziekenhuis-W Peter J Fontijne, Saskia C Wiersma, Bastiaan Boetes, Edgar JT ten Holder; Leids Universitair Medisch CentrumHuub JL van der Heide, Jochem Nagels, Enrike HMJ van der Linden-van der Zwaag; Medisch Centrum Haaglanden-Stefan B Keizer, Jan-Willem A Swen, Peter HC den Hollander, Bregje JW Thomassen; Onze Lieve Vrouwe Gasthuis -Rudolf W Poolman, Willem Jan Kleyn Molekamp, Frank RAJ de Meulemeester, Arthur EB Kleipool, Robert Haverlag, Maarten P Simons, Eduard LAR Mutsaerts; Ruwaard van Putten Ziekenhuis-Rob Kooijman, Roelf R Postema, René JTM Bleker, Harald IH Lampe; SlotervaartziekenhuisLein Schuman, John Cheung, Frank van Bommel, W Paul CA Winia, Daniel Haverkamp, Harm van der Vis; Spaarne Ziekenhuis_-Peter A Nolte, Michel PJ van den Bekerom, Tjitte de Jong, Arthur van Noort, Diederik A Vergroesen, Bernard G Schutte Tergooiziekenhuizen—Harm M van der Vis, Lijkele Beimers, Jasper de Vries, Arthur W Zurcher, GH Rob Albers, Maarten Rademakers, Stefan Breugem, Ibo van der Haven, Pieter Jan Damen, Gythe $\mathrm{H}$ Bulstra, Martin M Campo, Mathijs P Somford, Daniël Haverkamp.

International: The Alfred-Susan Liew, Harvinder Bedi, Ashley Carr, Andrew Chia, Steve Csongvay, Craig Donohue, Stephen Doig, Elton Edwards, Max Esser, Richard Freeman, Andrew Gong, Doug Li, Russell Miller, Lu Ton, Otis Wang, lan Young, Adam Dowrick, Zoe Murdoch, Claire Sage. Oslo University HospitaLFrede Frihagen, Lars Nordsletten, John Clarke-Jenssen, Geir Hjorthaug, Anne Christine Brekke, Elise Berg Vesterhus, Ringerike Sykehus Hospital-Ingunn Skaugrud. Hospital Universitario Costa del Sol-Enrique Guerado, Encarnacion Cruz, Juan Ramon Cano. Hospital Dr Josep TruetaMiguel Angel Froufe, Lluis Marull Serra, Samer Al-dirra, Cristina Martinez. The Geelong Hospital-Richard Page, David Bainbridge, Richard Angliss, Ben Miller, Andrew Thomson, Graeme Brown, Simon Williams, Kevin Eng, David Bowyer, John Skelley, Chatar Goyal, Sally Beattie. Ratandeep Hospital \& Research Centre, Kanpur- Pradeep Tripathi, Sandesh Katiyar, Preksha Shukla. Hospital de la Ribera_Francisco José Tarazona Santabalbina. Vall d'Hebron University Hospita —Ernesto Guerra-Farfán, Jordi Teixidor Serra, Jordi Tomas Hernandez, Marc Aguilar Garcia, Vicente Molero Garcia, Sergi Barrera, Jordi Selga, Teresa Sanclemente, Maria Villar, Joan Bago, Miriam Garrido. James Cook University HospitaL-Amar Rangan, Birgit Hanusch, Lucksy Kottam. North Shore Hospital-William Farrington, Carol Greene.

Contributors Writing Committee: MB, PJD, TAE, LT, EHS, KJK, FF, RWP, KT, EG-F, KM, SS, GHG. MB, PJD, TAE, LT, EHS, KJK, FF, RWP, KT, EG-F, KM, SS, and GHG made substantial contributions to conception and design, or acquisition of data, or analysis and interpretation of data. MB, PJD, TAE, LT, EHS, KJK, FF, RWP, KT, EG-F, KM, SS, and GHG have been involved in drafting the manuscript or revising it critically for important intellectual content. All authors read and approved the final manuscript. Steering Committee: MB, PJD, TAE, LT, EHS, KJK, FF, RWP, KT, EG-F, Stephen D Walter, GHG. Global Methods Centre: MB; SS (Research Programme Manager); KM, Paula McKay, Marilyn Swinton, Taryn Scott, (Project Management); Diane Heels-Ansdell, (Statistical Analysis); Lisa Buckingham, Aravin Duraikannan (Data Management; McMaster University). US Methods Centre: TAE; Heather Silva (Research Coordination; Boston University Medical Center). Netherlands Methods Centre: RWP, Martin J Heetveld; Esther MM Van Lieshout (Research Coordination), Paul TPW Burgers (Trial Coordination; Erasmus Medical Centre, Rotterdam). Central Adjudication Committee: MB, Robert D Zura, EHS, Victoria Avram, Ajay Manjoo, Dale Williams. Data Safety Monitoring Board (CIHR): John Antoniou, Tim Ramsay, Earl R Bogoch, Andrew Trenholm. Data Safety Monitoring Board (NIH): Stephen Lyman, Madhu Mazumdar, Kevin J Bozic, Mark Luborsky, Stuart Goodman, Susan Murray.

Funding Research grants were received from the following: Canadian Institutes of Health Research (CIHR; MB, GG and PJD) grant number MOP-126188, MOP-123609, National Institutes of Health (NIH; TAE) grant number 1UM1AR063386-01, 1R01AR055130-01A1, 5R01AR055130-02, ZorgOnderzoek Nederland-medische wetensehappen (ZonMw; EMM van Lieshout) grant number 170882503, Sophies Minde Foundation for Orthopaedic Research (L Nordsletten and FF), and McMaster Surgical Associates (MB) grant number 8-61107. MB was also funded, in part, by a 
Canada Research Chair in Musculoskeletal Trauma which is unrelated to the present study (McMaster University, Hamilton, ON, Canada).

Competing interests None.

Ethics approval McMaster University Research Ethics Board.

Provenance and peer review Not commissioned; externally peer reviewed.

Open Access This is an Open Access article distributed in accordance with the Creative Commons Attribution Non Commercial (CC BY-NC 4.0) license, which permits others to distribute, remix, adapt, build upon this work noncommercially, and license their derivative works on different terms, provided the original work is properly cited and the use is non-commercial. See: http:// creativecommons.org/licenses/by-nc/4.0/

\section{REFERENCES}

1. Cooper C, Campion G, Melton LJ, III. Hip fractures in the elderly: a world-wide projection. Osteoporos Int 1992;2:285-9.

2. Dhanwal DK, Dennison EM, Harvey NC, et al. Epidemiology of hip fracture: worldwide geographic variation. Indian J Orthop 2011:45:15-22.

3. Johnell O, Kanis JA. An estimate of the worldwide prevalence, mortality and disability associated with hip fracture. Osteoporos Int 2004; 15:897-902

4. Iorio R, Schwartz B, Macaulay W, et al. Surgical treatment of displaced femoral neck fractures in the elderly: a survey of the American association of hip and knee surgeons. Arthrop 2006;21:1124-33.

5. Cummings SR, Rubin SM, Black $D$. The future of hip fractures in the United States. Numbers, costs, and potential effects of postmenopausal estrogen. Clin Orthop Relat Res 1990;252:163-6.

6. Papadimitropoulos EA, Coyte PC, Josse RG, et al. Current and projected rates of hip fracture in Canada. CMAJ 1997;157:1357-63.

7. American Academy of Orthopaedic Surgeons. AAOS urges hip fracture reform. Am Acad Orthop Surg Bull 1999;47. http://www2. aaos.org/aaos/archives/bulletin/aug99/acd nw11.htm

8. Moran CG, Wenn RT, Sikand M, et al. Early mortality after hip fracture: is delay before surgery important? J Bone Joint Surg 2005;87:483-9.

9. Jongjit J, Komsopapong L, Songjakkaew $\mathrm{P}$, et al. Health-related quality of life after hip fracture in the elderly community-dwelling Southeast Asian J Trop Med Public Health 2003;34:670-4.

10. Carrière $\mathrm{G}$. Hip fracture outcomes in the household population. Health Rep 2007;18:37-42.

11. Hopley C, Stengel D, Ekkernkamp A, et al. Primary total hip arthroplasty versus hemiarthroplasty for displaced intracapsular hip fractures in older patients: systematic review. BMJ 2010;340:c2332.

12. Liao L, Zhao Jm, Su W, et al. A meta-analysis of total hip arthroplasty and hemiarthroplasty outcomes for displaced femoral neck fractures. Arch Orthop Trauma Surg 2012;132:1021-9.

13. Burgers PTPW, Van Geene AR, Van den Bekerom MPJ, et al. Total hip arthroplasty versus hemiarthroplasty for displaced femoral neck fractures in the healthy elderly: a meta-analysis and systematic review of randomized trials. Int Orthop 2012;36:1549-60.

14. Bhandari M, Devereaux PJ, Tornetta P III, et al. Operative management of displaced femoral neck fractures in elderly patients. An international survey. J Bone Joint Surg Am 2005;87:2122-30.

15. Pandis N. Randomization. Part 2: minimization. Am J Orthod Dentofacial Orthop 2011;140:902-4.

16. Gillespie WJ. Extracts from "clinical evidence": hip fracture. $B M J$ 2001;322:968-75.

17. McHorney CA, Ware JE Jr, Rogers W, et al. The validity and relative precision of MOS short- and long-form health status scales and Dartmouth COOP charts. Results from the medical outcomes study. Med Care 1992;30(Suppl 5):MS253-65

18. Bellamy N, Buchanan WW, Goldsmith $\mathrm{CH}$, et al. Validation of WOMAC: a health status instrument for measuring clinically important patient relevant outcomes to antirheumatic drug therapy in patients with osteoarthritis of the hip or knee. J Rheumatol 1988; $15: 1833-40$

19. Brooks R, Rabin RE, de Charro F, eds. The measurement and valuation of health status using EQ-5D: a European perspective. Dordrecht, The Netherlands: Kulwer Academic Publishers, 2003.

20. Podsiadlo D, Richardson S. The timed 'up and go' test: a test of basic functional mobility for frail elderly persons. J Am Geri Soc 1991;39:142-8
21. Ware JE, Kosinski M, Keller SD. A 12-item short-form health survey: construction of scales and preliminary tests of reliability and validity. Med Care 1996;34:220-33.

22. Dattani R, Slobogean GP, O'Brien PJ, et al. Psychometric analysis of measuring functional outcomes in tibial plateau fractures using the Short Form 36 (SF-36), Short Musculoskeletal Function Assessment (SMFA) and the Western Ontario McMaster Osteoarthritis (WOMAC) questionnaires. Injury 2013;44:825-9.

23. Laflamme GY, Rouleau DM, Leduc S, et al. The Timed Up and Go test is an early predictor of functional outcome after hemiarthroplasty for femoral neck fracture. J Bone Joint Surg Am 2012;94:1175-9.

24. McMeeken J, Stillman B, Story I, et al. The effects of knee extensor and flexor muscle training on the timed-up-and-go test in individuals with rheumatoid arthritis. Physiother Res Int 1999;4:55-67.

25. Shumway-Cook A, Brauer S, Woollacott M. Predicting the probability for falls in community-dwelling older adults using the Timed Up \& Go Test. Phys Ther 2000;80:896-903.

26. Ravikumar KJ, Marsh G. Internal fixation versus hemiarthroplasty versus total hip arthroplasty for displaced subcapital fractures of femur-13 year results of a prospective randomised study. Injury 2000;31:793-7.

27. Callaghan JJ, Heekin RD, Savory CG, et al. Evaluation of the learning curve associated with uncemented primary porous-coated anatomic total hip arthroplasty. Clin Orthop Relat Res 1992;282:132-44.

28. Ellinor N, Lindelof N, Rosendahl $\mathrm{E}$, et al. Prognostic validity of the Timed Up-and-Go test, staff's global judgement and fall history in evaluating fall risk in residential care facilities. Age Ageing 2008;37:442-8.

29. Katz JN, Losina E, Barrett J, et al. Association between hospital and surgeon procedure volume and outcomes of total hip replacement in the United States medicare population. J Bone Joint Surg Am 2001;83-A:1622-9.

30. Devereaux PJ, Bhandari M, Clarke $M$, et al. Need for expertise based randomised controlled trials. BMJ 2005;330:88-91.

31. van den Bekerom MP, Hilverdink EF, Sierevelt IN, et al. A comparison of hemiarthroplasty with total hip replacement for displaced intracapsular fracture of the femoral neck: a randomised controlled multicentre trial in patients aged 70 years and over $J$ Bone Joint Surg Br 2010;92:1422-8.

32. Baker RP, Squires B, Gargan MF, et al. Total hip arthroplasty and hemiarthroplasty in mobile, independent patients with a displaced intracapsular fracture of the femoral neck. A randomized, controlled trial. J Bone Joint Surg Am 2006;88:2583-9.

33. Blomfeldt $\mathrm{R}$, Törnkvist $\mathrm{H}$, Eriksson $\mathrm{K}$, et al. A randomised controlled trial comparing bipolar hemiarthroplasty with total hip replacement for displaced intracapsular fractures of the femoral neck in elderly patients. J Bone Joint Surg Br 2007:89:160-5.

34. Keating JF, Grant A, Masson M, et al. Displaced intracapsular hip fractures in fit, older people: a randomised comparison of reduction and fixation, bipolar hemiarthroplasty and total hip arthroplasty. Health Technol Assess 2005;9:1-65.

35. Macaulay W, Nellans KW, Garvin KL, et al. Prospective randomized clinical trial comparing hemiarthroplasty to total hip arthroplasty in the treatment of displaced femoral neck fractures. J Arthroplasty 2008;23(6 Suppl 1):2-8.

36. Collett D. Modelling survival data in medical research. London, UK: Chapman \& Hall, 1994.

37. Cohen J. Statistical power analysis for the behavioral sciences. 2nd edn. Hillsdale, NJ: Lawrence Erlbaum Associates, 1988.

38. Ware JE, Sherbourne CD. The MOS 36-item short form health survey (SF-36). I. Conceptual framework and item selection. Med Care 1992;30:473-83.

39. Jaglal S, Lakhani Z, Schatzker J. Reliability, validity, and responsiveness of the lower extremity measure for patients with a hip fracture. J Bone Joint Surg Am 2000;82:955-62.

40. Tubach F, Ravaud P, Baron G, et al. Evaluation of clinically relevant changes in patient reported outcomes in knee and hip osteoarthritis: The minimal clinically important improvement. Ann Rheum Dis 2005;64:29-33.

41. Tidermark J, Ponzer S, Svensson O, et al. Internal fixation compared with total hip replacement for displaced femoral neck fractures in the elderly: a randomised, controlled trial. $J$ Bone Joint Surg $\mathrm{Br}$ 2003;85:380-8.

42. Montori VM, Devereaux PJ, Adhikari NK, et al. Stopping randomized clinical trials early for benefit: a protocol of the study of trial policy of interim truncation-2 (STOPIT-2). Trials 2009;10:49.

43. Fine J, Gray R. A proportional hazards model for the sub-distribution of a competing risk. J Am Stat Assoc 1999;94:496-509. 